Research Paper

\title{
Up-regulated FHLI Expression Maybe Involved in the Prognosis of Hirschsprung's Disease
}

\author{
Li-Li Wang, Hui Gu, Yang Fan, Yi Zhang, Di Wu, Jia-Ning Miao, Tian-Chu Huang, Hui Li, Zheng-Wei \\ Yuan $\bowtie$
}

Key laboratory of Health Ministry for Congenital Malformation, Shengjing Hospital, China Medical University, Shen yang, 110004, P.R. China

$\triangle$ Corresponding author: Dr. Zheng-Wei Yuan. No. 36, Sanhao Street, Heping District, Shen yang, China, 110004. Key Laboratory of Health Ministry for Congenital Malformation, Shengjing Hospital, China Medical University, Shenyang, 110004, P.R. China. Tel: +86-24-23929903; Fax: +86-24-23929903; E-mail: yuanzw@hotmail.com

(c) Ivyspring International Publisher. This is an open-access article distributed under the terms of the Creative Commons License (http://creativecommons.org/ licenses/by-nc-nd/3.0/). Reproduction is permitted for personal, noncommercial use, provided that the article is in whole, unmodified, and properly cited.

Received: 2013.07.29; Accepted: 2013.12.02; Published: 20I4.0I.20

\begin{abstract}
Background: In a subset of patients with Hirschsprung's disease (HSCR), gastrointestinal motor dysfunction persisted long after surgical correction. Gastrointestinal motility is achieved through the coordinated activity of the enteric nervous system, interstitial cells of Cajal, and smooth muscle (SMC) cells. Inhibition of four-and-a-half LIM protein-I (FhlI) expression by siRNA significantly decreases pulmonary artery SMCs migration and proliferation. Furthermore when up-expressing $\mathrm{FHLI}$ in atrial myocytes, $\mathrm{K}(+)$ current density markedly increases, therefore changing myocytes' response to an electrical stimulus. However whether FHLI in colon SMCs (the final effector organ) influences intestinal motility in HSCR patients has not been clarified. Methods: FHLI mRNA and protein expressions were analyzed in $32 \mathrm{HSCR}$ colons and 4 normal colons. Results: Smooth muscle layers were thicken and disorganized in HSCR. FHLI was expressed in the ganglion cells of the myenteric plexus, submucosa, as well as in the longitudinal and circular muscle layer of the ganglionic colon. FHLI mRNA relative expression level in aganglionic colons was I.06 \pm 0.49 (ganglionic colon relative expression level was I) $(P=0.44)$. FHLI protein gray level relative to GAPDH in normal colons was $0.83 \pm 0.09$. FHLI expression level in ganglionic colon $(I .66 \pm 0.30)$ or aganglionic colon $(I .8 I \pm 0.35)$ was significantly higher than that in normal colons ( $P=0.045$ and $P=0.04 \mathrm{I}$, respectively). Meanwhile, we found $\mathrm{FHLI}$ expression in aganglionic colon was slightly stronger than that in ganglionic colon $(P=0.036)$. Conclusion: These data suggested that up-regulated FHLI in smooth muscle in HSCR might be associated with intestinal wall remodeling in HSCR and might be one of the risk factors for gastrointestinal motor dysfunction.
\end{abstract}

Key words: FHL1; Hirschsprung's disease; expression; smooth muscle; prognosis

\section{Introduction}

Hirschsprung's disease (HSCR, aganglionic megacolon, OMIM 142623) is a developmental disorder characterized by congenital absence of intrinsic ganglion cells in the myenteric (Auerbach) and submucosal (Meissner) plexuses of the gastrointestinal tract, leading to tonic contraction of the affected segment, intestinal obstruction, and massive distension of the bowel [1]. Resection of the abnormally inner- vated bowel is essential to avoid life threatening complications. Previous data showed despite successful surgical treatment, there was a large proportion of patients in whom constipation, soiling and abdominal pain persisted. After an average of 9 years, $53 \%$ of patients continued to suffer from fecal incontinence and $22 \%$ were still constipated [2-4]. The reasons for these persistent symptoms were not clear, 
although data were emerging to suggest that those with constipation have a neuropathy proximal to the aganglionic segment [5]. Aganglionosis is attributed to a failure in the time-specific migration of enteric neural crest-derived cells into the intestinal tract. Most studies on the pathogensis of HSCR were concerned on the autologous abnormity of migrating enteric neural precursors (ENPs), and the results showed that mutations of the RET, GDNF, EDNRB, SOX10, NRG1, NKX2-1 and EDN3 genes appeared to give dominant, recessive, or polygenic patterns of inheritance [6-9]. However, research on target cells such as smooth muscle was rather limited. Smooth muscle thickening and intestinal wall remodeling existed in aganglionic and ganglionic segment of HSCR. But the influence of intestinal wall remodeling on prognosis of Hirschsprung disease, and the molecular pathways triggering the thickening and remodeling process are still poorly understood.

Previous studies indicated that smooth muscle had a major impact on determining the number of innervating neurons. Jacob CL found that smooth muscle of the aganglionic colon was less favourable for neuronal development than that of normal colon and the mechanism was not clear [10]. Many proteins with abnormal function or expression level in the smooth muscle cells of the aganglionic part of the colon in HSCR have been identified, such as cholinoreceptors, alpha-smooth muscle isoactin, Semaphorin 3A, sarcoglycan subcomplex and Connexin 43 etc [11- 17]. These proteins participate in the pathogenesis through impairing intercellular communication between interstitial cells of Cajal and smooth muscle cells or altering the cytoskeleton in smooth muscle cells or disturbing the microenvironment around the targets of migrating neural crest cells in autocrine or paracrine manner during colon development. But the mechanism of the proteins in smooth muscle participated in intestinal dysfunction in HSCR patients has not been clarified.

The FHL1 gene, located on chromosome Xq27.2, encodes four-and-a-half LIM protein-1 (FHL1) and its spliced isoform, SLIMMER or FHL1C. Fhl1 protein contains four and a half tandemly repeated LIM domains and Northern blot analysis confirms strikingly high expression of Fhl1 in skeletal muscle and heart, with considerably lower expression levels observed in several other tissues, including colon, small intestine, and prostate $[18,19]$. Fhl1 can also promote myoblast spreading and migration by inhibiting integrin-mediated myoblast adhesion [20]. FHL1 mutations have been identified in a spectrum of human skeletal and cardiac muscle diseases [21-24]. In rat aortic smooth muscle cells (SMCs) FHL1 knockdown can significantly inhibit the proliferation but exert no significant effect on cell apoptosis [25]. Kwapiszewska $\mathrm{G}$ demonstrated that inhibition of Fhl1 expression by siRNA significantly decreased pulmonary artery SMCs migration and proliferation, so these results suggested Fhl1 was the key factor triggering the vascular remodeling process in pulmonary hypertension [26]. However, the functions of FHL1 in colon SMCs and its role in the HSCR have not been characterized in studies.

\section{Methods}

\section{Patients and controls}

Colon tissues from 32 sporadic HSCR patients, aged from one month to seven years, were obtained from Shengjing Hospital, China Medical University. HSCR diagnosis was based on histological examination of surgical resection for absence of enteric plexuses. Ganglionic colon in HSCR was the most rostral part of the colon that was surgically removed from patients. In addition there were 4 colons from newborn infants, died from non-nervous or digestive system diseases. The study was approved by the local ethical committee and all the subjects involved in the study gave written informed consent.

\section{Immunohistochemistry}

Sections were deparaffinized in xylene, hydrated and incubated with $3 \% \mathrm{H}_{2} \mathrm{O}_{2}$ in methanol for $30 \mathrm{~min}$ at room temperature to block endogenous peroxidase, then washed twice in PBS $(2 \times 5 \mathrm{~min})$ and incubated in normal serum for $30 \mathrm{~min}$ at room temperature to block non-specific sites. Sections were incubated overnight at $4{ }^{\circ} \mathrm{C}$ with the primary antibody against FHL1 (Santa Cruz, California, USA; polyclonal goat, sc-23175) at a concentration of $1 \mu \mathrm{g} / \mathrm{ml}$, washed twice with PBS ( $2 \times 15 \mathrm{~min} \mathrm{~min})$; then transferred to $1: 200 \mathrm{v} / \mathrm{v}$ biotinylated IgG anti-goat serum in PBS for $60 \mathrm{~min}$ at room temperature; washed twice with PBS $(2 \times 5 \mathrm{~min})$; incubated again in ABC Elite reagent in PBS for $30 \mathrm{~min}$ at room temperature; washed twice with $\mathrm{PBS}(3 \times 5$ min); took the final incubation in $0.02 \% \mathrm{H}_{2} \mathrm{O}_{2}$ and $0.075 \%$ diaminobenzidine in $0.05 \mathrm{M}$ Tris buffer $(\mathrm{pH}$ 7.6), kept for 1min in a dark room; and rinsed in distilled water. Negative controls were obtained in each instance by omitting the primary antibody.

\section{Real-time PCR reaction}

FHL1 gene expression in HSCR patients were detected using SYBR-Green I real-time PCR. RNA from aganglionic and ganglionic colon tissue of 32 HSCR patients were extracted using the TRIzol Reagent (Invitrogen, California, USA) according to the manufacturer's protocol. cDNA synthesis was performed starting from $3 \mu \mathrm{g}$ of RNA using the TaKaRa RNA PCR kit (Takara, Dalian, JAPAN). 
Real-time PCR amplifications were performed in triplicates on Light Cycle (Roche, Basel, Switzerland) using the following oligonucleotides: FHL1-1:5'-GTA GTCGTGCCAGGATTGT-3'; FHL1-2:5'-GCTGTGGA GGACCAGTATTA-3' (product size $=142 \mathrm{bp}$ ). The housekeeping gene GAPDH (Takara DR3702) was used as an endogenous control. The relative levels of FHL1 gene expression for each sample were calculated using the $2^{-\Delta \Delta c t}$ method.

\section{Western-blot}

Antibodies against FHL1 were purchased from Sigma-Alorich (Sigma-Alorich, Saint Louis, USA; monoclonal mouse, WH0002273M1). Aganglionic and ganglionic colon segments of HSCR samples and colon segments of newborn infants were frozen and lysed in buffer. The protein concentration of each lysate was determined using the bicinchoninic acid (BCA) kit according to the manufacture's protocol. Total protein $(90 \mu \mathrm{g})$ was applied to each lane on $12 \%$ SDS-polyacrylamide gels. After electrophoresis, the polyvinylidene fluoride (PVDF) membranes were washed in Tris-buffered saline containing $0.1 \%$ Tween-20, and then incubated with primary antibody (diluted 1:2000) followed by secondary antibody (diluted 1:2000). Immunostained bands were detected with a ProtoBlot II AP System with a stabilized sub- strate (Promega, Madison, USA). GAPDH protein was used as internal control.

\section{Statistical analysis}

FHL1 expression values are expressed as mean $\pm S E M$. Data were analyzed with Student's T test. $P$ values less than 0.05 were considered to be statistically significant.

\section{Results}

\section{Immunostaining of FHLI in HSCR patients}

The HE and immunostaining of FHL1 in 4 HSCR colons and 4 normal colons were accomplished. Circular muscle layer and longitudinal muscle layer were thickening at different extent in aganglionic and ganglionic segment of HSCR. Compared with normal colon the arrangement of circular muscle layer in aganglionic segment of HSCR was disorganized (Fig.1). Immunohistologic study revealed that in the ganglionic segment of HSCR, FHL1 was expressed in the ganglia cells in myenteric, submucosa, circular muscle layer and longitudinal muscle layer. However in the aganglionic segment of HSCR we found expression levels of FHL1 in the circular muscle layer, submucosa, and longitudinal muscle layer (Fig.1).
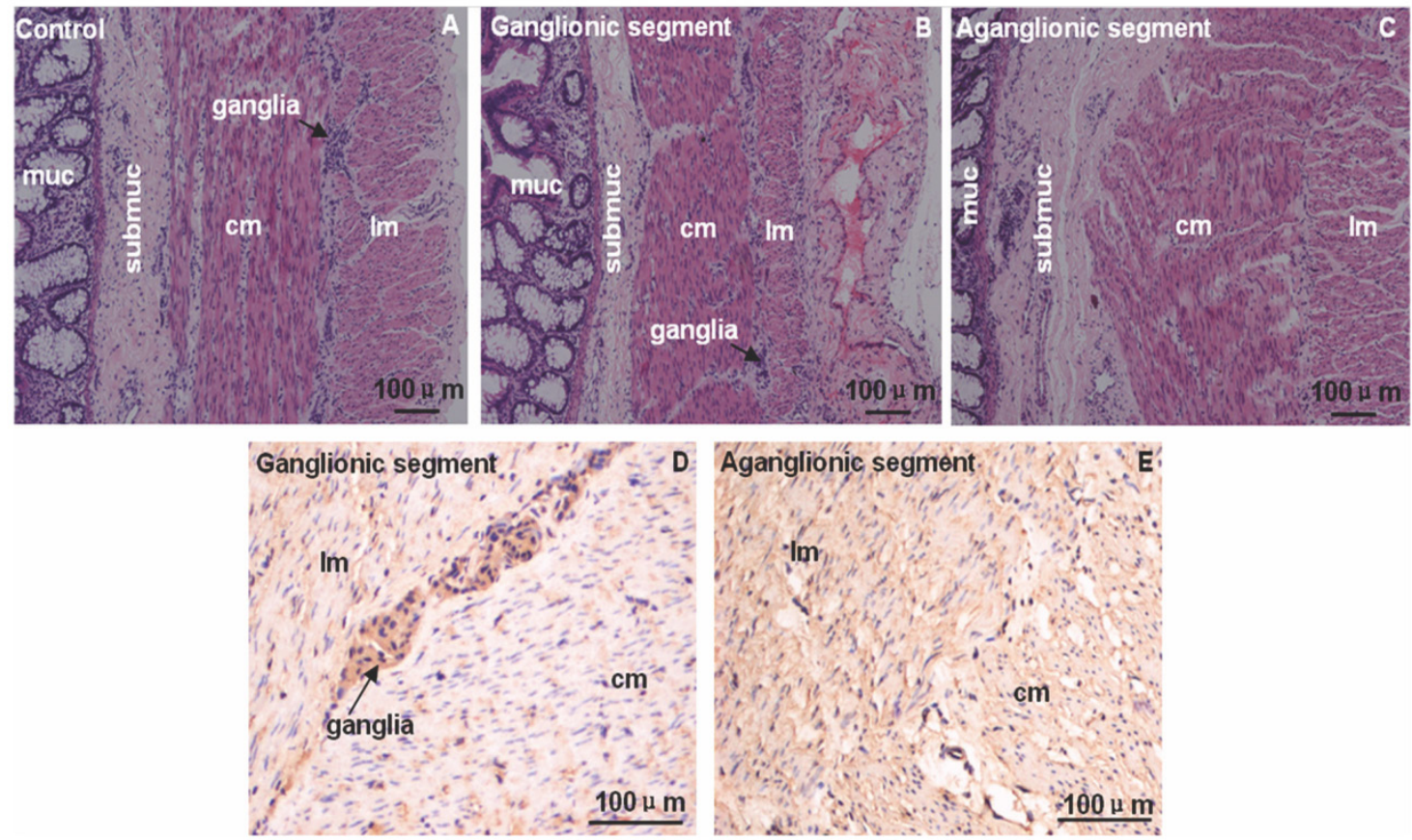

Figure I. Immunol staining of FHLI in colon. A-C: HE staining in normal colon, ganglionic segment and aganglionic segment of HSCR. D-E: FHLI staining illustrated that the expression was restricted to the ganglia cells in the myenteric, submucosa, longitudinal muscle and circular muscle layer in ganglionic segment. Furthermore stronger FHLI signal was found in the circular muscle layer and longitudinal muscle in HSCR aganglionic segment. The arrow denoted ganglia cells. muc: mucosa; submuc: submucosa; Im: longitudinal muscle; cm: circular muscle. Scale bar: $100 \mu \mathrm{m}$ 


\section{FHLI gene expression in HSCR patients}

FHL1 mRNA and protein expressions were analyzed in 32 HSCR patients and 4 normal colons. As revealed in Fig 2, the FHL1 mRNA relative expression in aganglionic colons was 1.06 \pm 0.49 (ganglionic colon relative expression level was 1$)(\mathrm{P}=0.44)$. FHL1 protein gray level relative to GAPDH in normal colons was $0.83 \pm 0.09$. FHL1 expression level in ganglionic colon $(1.66 \pm 0.30)$ or aganglionic colon $(1.81 \pm 0.35)$ was significantly higher than that in normal colons ( $P=0.045$ and $P=0.041$, respectively). Meanwhile, we found FHL1 expression in aganglionic colon was slightly stronger than that in ganglionic colon $(P=0.036)$, as shown in Fig 3.

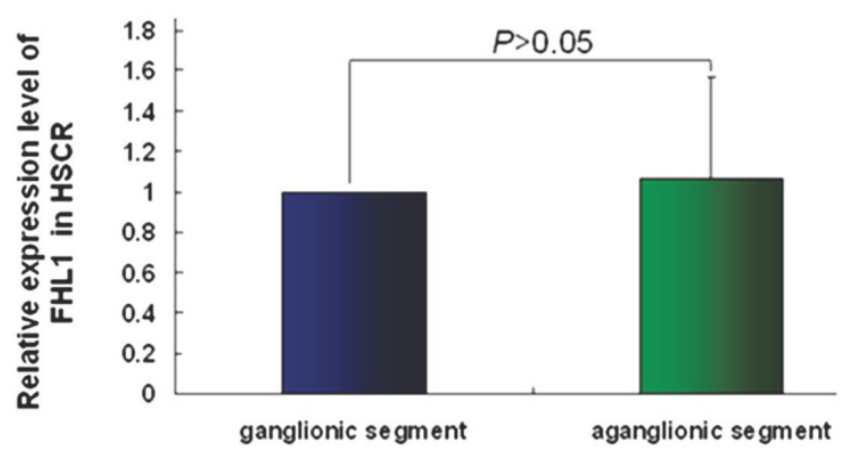

Figure 2. The relative expression level of FHLI mRNA in HSCR colon.

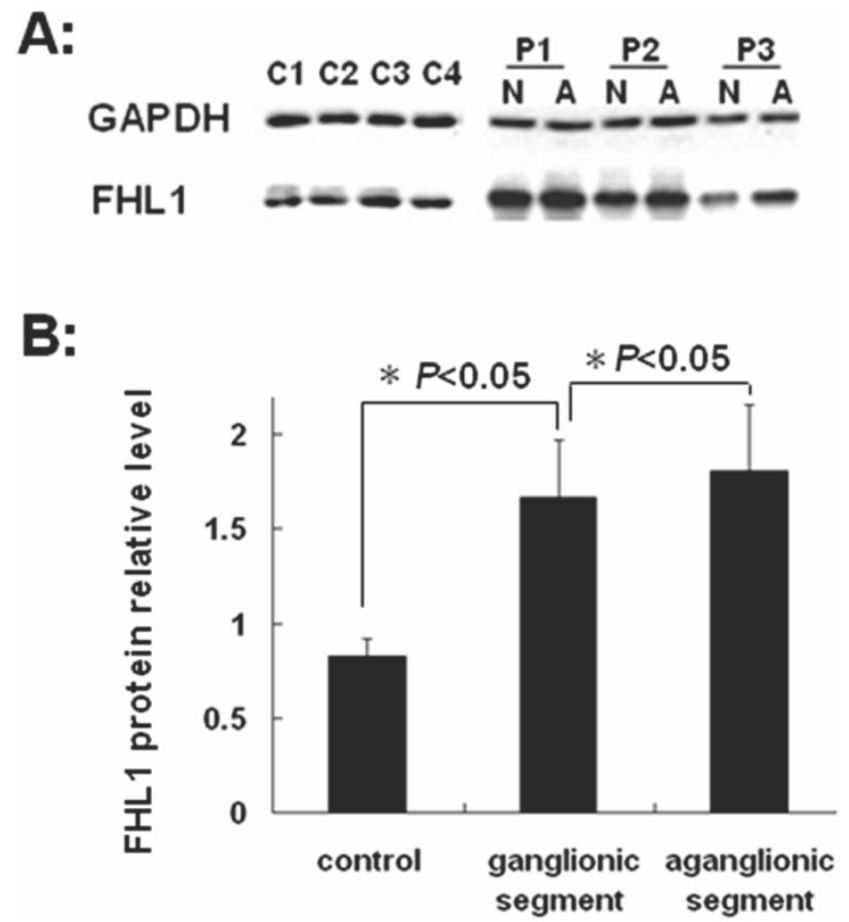

Figure 3. Western-blot of FHLI in colon $\mathbf{A}$ : Western-blot of FHLI in HSCR colon and normal colons. PI,2,3: Patient I,2,3; N: ganglionic segment of colon in HSCR; $\mathrm{A}$ : aganglionic segment of colon in HSCR; $\mathrm{CI}, 2,3,4$ : Control I,2,3,4; B: The gray analysis of Western-blot using SynGene Gene Tools gel analysis program.

\section{Discussion}

In patients with Hirschsprung disease (HSCR), resection of the affected aganglionic bowel segment is the accepted treatment but constipation, soiling and abdominal pain are the challenging problems in some children after pull-through surgery for Hirschsprung disease (HSCR). Data showed that, in a subset of patients with HSCR, gastrointestinal motor dysfunction persisted long after surgical correction [2, 27]. The physiology underlying the persistent symptoms in children after surgery for Hirschsprung's disease is yet not clear.

Gastrointestinal motility is achieved through the coordinated activity of enteric nervous system (ENS), interstitial cells of Cajal (ICC), and smooth muscle (SM) cells. ICC and ENS supply SM cells with the necessary stimuli to contract and generate motility. The response of SM cells to an electrical stimulus provided by the neighboring ICC network is mediated by the activation of a wide variety of voltage-dependent ion channels within their cell membrane. Voltage-activated ion channels expressed in ICC and smooth muscle cells, such as $\mathrm{Ca}^{2+}$ channels, $\mathrm{Na}^{+}$channels or $\mathrm{K}^{+}$channels, are contributed to the electrical activity and subsequent contractile activity of intestinal smooth muscle [28-30].

A decreased number of c-kit positive cells (interstitial cells of Cajal) in the normoganglionic segment is regarded as a clue to predict a poor clinical outcome after surgery, probably due to poor intestinal motility [31, 32]. Smooth muscle thickening and intestinal wall remodeling exist in aganglionic and ganglionic segment of HSCR. An additional enteric smooth muscle layer was firstly reported in a patient with Mowat-Wilson syndrome and Hirschsprung's disease. After the resection of the aganglionic colon at the age of 5 months, this patient started to suffer from intermittent constipation. Although the exact mechanism of abnormal gut motility in this case was unknown, the supernumerary muscle and its associated neural plexus might be responsible for the patient's late complication [33]. In addition, impairment of cytoskeleton in SMC of aganglionic bowel may be associated with abnormal gut motility. Since cytoskeletal proteins are required for the coordinated contraction of muscle cells, their absence or notable reduction in the aganglionic bowel of HSCR may be responsible for the motility dysfunction in the aganglionic segment [34]. Sarcoglycan subcomplex (SG) support the development and maintenance of muscle cells, therefore up-regulation of a-Sarcoglycan subcomplex in SMC is probably an acquired phenomenon relating to the intestinal dysmotility which persists in $20 \%$ of patients after resection of the aganglionic bowel [14]. But whether or not other proteins in smooth muscle 
participate in intestinal dysfunction in HSCR patients is not clear.

In this study we found that circular muscle layer and longitudinal muscle layer were thickening at different extent in aganglionic and ganglionic segment of HSCR and the arrangement of circular muscle layer in aganglionic segment of HSCR was disorganized (Fig.1). FHL1 was expressed in the ganglion cells of the myenteric plexus, submucosa, as well as in the longitudinal and circular muscle layer of the ganglionic colon. FHL1 expression level in ganglionic colon or aganglionic colon was significantly higher than that in normal colons. Meanwhile, we found FHL1 expression in aganglionic colon was slightly stronger than that in ganglionic colon.

Four and a half LIM domains protein 1 (FHL1) is the most widely expressed member of the FHL family of proteins, consisting of four and a half highly conserved LIM domains [35]. LIM domains have been observed to act as modular protein-binding interfaces mediating protein-protein interactions in the cytoplasm and the nucleus [36]. Northern blot analysis confirmed strikingly high expression of Fhl1 in skeletal muscle and heart, with considerably lower expression levels observed in several other tissues, including the colon, small intestine, and prostate. FHL1 mutations have been identified in a spectrum of human skeletal and cardiac muscle diseases [21-24]. FHL1 could alter cytoskeleton and cell shape by binding with PDZ and LIM domain protein 1 (PDLIM1), Gelsolin (GSN), gamma-actin (ACTG) and a-actin (ACTN1) $[37,38]$. In rat aortic smooth muscle cells (SMCs) FHL1 knockdown could significantly inhibit the proliferation of SMCs but exerted no significant effect on cell apoptosis [25]. Kwapiszewska G demonstrated that inhibition of Fhl-1 expression by siRNA significantly decreased pulmonary artery SMCs migration and proliferation, suggesting that Fhl1 was the key factor triggering the vascular remodeling process in pulmonary hypertension [26]. Protein-protein interactions were critical for the normal membrane trafficking, localization, and function of voltage-gated ion channels. Immunoprecipitation experiments confirmed a physical interaction of FHL1 with the $\mathrm{K}(+)$ channel (KCNA5) complex in human atrium. With coexpression of FHL1, K (+) current density was markedly increased in atrial myocytes $[39,40]$.

Data suggested that the alteration of delayed rectifier $\left(\mathrm{I}_{\mathrm{K}}\right) \mathrm{K}^{+}$current and Kv1.2 expression in DRG neurons from Irritable bowel syndrome (IBS) model rats represented a molecular mechanism underlying visceral pain and hyperexcitability in IBS [41]. In the study of chronic stress-induced colonic hypermotility, Ying Liu found that repeated water avoidance stress
(WAS) treatment resulted in up-regulation of Kir6.1 and SUR2B of $K_{\text {ATP }}$ channels in the colon devoid of mucosa and submucosa [42]. These results demonstrated that $\mathrm{Kv}$ channels in the DRG neurons or colonic smooth muscle cells were associated with gastrointestinal motility and might have potential clinical utility in treating gastrointestinal motility disorders.

However whether FHL1 in smooth muscle (SM) cells (the final effector organ) influences intestinal motility in HSCR patients or its mechanism in this process has not been clarified. Our results and all the above-mentioned data suggested that up-regulated expression level of FHL1 in ganglionic colon or aganglionic colon in HSCR might be associated with smooth muscle thickening and intestinal wall remodeling in HSCR through increasing the proliferation of smooth muscle cell or altering its skeleton. Furthermore when up-expressed FHL1 in smooth muscle cell, $\mathrm{K}(+)$ current density would also be markedly increased, like in atrial myocytes, thus changing their response to an electrical stimulus provided by the neighboring ICC. In summary we hypothesize that FHL1 in smooth muscle (SM) cells (the final effector organ) could influence intestinal motility in HSCR patients by remodeling intestinal wall and changing their electrical response to stimulus provided by the neighboring ICC. Moreover since this experiment revealed that FHL1 expression in ganglionic colon, reserved in the operation, was much higher than that in the normal colon from newborn infants died from non-nervous or digestive system diseases, we believed that molecular abnormity also existed in this relatively normal colon in HSCR. So after resection of the affected aganglionic bowel segment, constipation, soiling and abdominal pain (intestinal dysfunction) are the subsequent problems in some children after pull-through surgery for Hirschsprung's disease (HSCR). In further research we intend to screen gene abnormity in ganglionic colon in HSCR and investigate the mechanism of intestinal dysfunction existing after surgery which will provide a fresh clue for clinical therapy for HSCR.

\section{Acknowledgments}

This study was supported by National Natural Science Foundation of China (81070538, 81100434 and 81200481), Specialized Research Fund for the Doctoral Program of Higher Education (20102104120014, 20092104120010) and Scientific Research Fund of Liaoning Provincial Education Department (L2010637). We would like to express our gratitude to all the subjects who participated in the study.

\section{Abbreviations}

HSCR: Hirschsprung's Disease; SMC: smooth 
muscle cells; FHL1: four-and-a-half LIM protein-1; ENPs: enteric neural precursors; ENS: enteric nervous system; ICC: interstitial cells of Cajal; PDLIM1: PDZ and LIM domain protein 1; GSN: Gelsolin; ACTN1: a-actin.

\section{Competing Interests}

The authors have declared that no competing interest exists.

\section{References}

1. Angrist M, Kauffman E, Slaugenhaupt SA, et al. A gene for Hirschsprung disease (megacolon) in the pericentromeric region of human chromosome 10. Nat Genet. 1993; 4 :351-356.

2. Di Lorenzo C, Solzi GF, Flores AF, et al. Colonic motility after surgery for Hirschsprung's disease. Am J Gastroenterol. 2000;95:1759-1764.

3. Shono K, Hutson JM. The treatment and postoperative complications of Hirschsprung's disease. Pediatr Surg Int. 1994; 9:362-365.

4. Catto-Smith AG, Coffey CM, Nolan TM, et al. Fecal incon-tinence after the surgical treatment of Hirschsprung's disease. J Pediatr. 1995; 127:954-957.

5. Kobayashi H, Hirakawa $H$, Surana $R$, et al. Intestinal neuronal dysplasia is a possible cause of persistent bowel symptoms after pull-through operation for Hirschsprung's disease. J Pediatr Surg.1995;30:253-259.

6. Romeo G, Ronchetto P, Luo Y, et al. Point mutations affecting the tyrosine kinase domain of the RET proto-oncogene in Hirschsprung's disease. Nature. 1994;367:377-378.

7. Garcia-Barceló MM, Lau DK, Ngan ES, et al. Evaluation of the NK2 homeobox 1 gene (NKX2-1) as a Hirschsprung's disease locus. Ann Hum Genet.2008; 72:170-177.

8. Garcia-Barcelo MM, Tang CS, Ngan ES, et al. Genome-wide association study identifies NRG1 as a susceptibility locus for Hirschsprung's disease. Proc Natl Acad Sci USA.2009; 106:2694-2699.

9. Walters LC, Cantrell VA, Weller KP, et al. Genetic Background Impacts Development Potential of Enteric Neural Crest-Derived Progenitors in the Sox10Dom Model of Hirschsprung Disease. Hum Mol Genet. 2010;19:4353-4372

10. Langer JC, Betti PA, Blennerhassett MG. Smooth muscle from aganglionic bowel in Hirschsprung's disease impairs neuronal development in vitro. Cell Tissue Res. 1994; 276:181-186.

11. Atanassova E, Todorov S, Noeva A, et al. Changes in the responsiveness of the colonic smooth muscle to carbachol in Hirschsprung's disease. Acta Physiol Pharmacol Bulg. 1991;17:19-24.

12. Fromont-Hankard G, Lafer D, Masood S. Altered expression of alpha-smooth muscle isoactin in Hirschsprung's disease. Arch Pathol Lab Med. 1996;120:270-274.

13. Nemeth L, Maddur S, Puri P. Immunolocalization of the gap junction protein Connexin43 in the interstitial cells of Cajal in the normal and Hirschsprung's disease bowel. J Pediatr Surg. 2000:35:823-828.

14. Arena S, Cutroneo G, Favaloro A, et al. Abnormal distribution of sarcoglycan subcomplex in colonic smooth muscle cells of aganglionic bowel. Int J Mol Med. 2010;25:353-359.

15. Wang LL, Fan Y, Zhou FH, et al. Semaphorin 3A expression in the colon of Hirschsprung disease. Birth Defects Res A Clin Mol Teratol.2011;91:842-847.

16. Wang LL, Zhang Y, Fan Y, et al. SEMA3A rs7804122 polymorphism is associated with Hirschsprung disease in the Northeastern region of China. Birth Defects Res A Clin Mol Teratol. 2012;94:91-95.

17. Jiang $Q$, Turner T, Sosa MX, et al. Rapid and efficient human mutation detection using a bench-top next-generation DNA sequencer. Hum Mutat. 2012;33:281-289.

18. Lee SM, Tsui SK, Chan KK, et al. Chromosomal mapping, tissue distribution and cDNA sequence of four-and-a-half LIM domain protein 1 (FHL1). Gene. 1998; 216:163-170.

19. Greene WK, Baker E, Rabbitts TH, et al. Genomic structure, tissue expression and chromosomal location of the LIM-only gene, SLIM1. Gene. 1999; 232:203-207.

20. Robinson PA, Brown S, McGrath MJ, et al. Skeletal muscle LIM protein 1 regulates integrin-mediated myoblast adhesion, spreading, and migration. Am J Physiol Cell Physiol. 2003;284:681-695.

21. Windpassinger $\mathrm{C}$, Schoser $\mathrm{B}$, Straub V, et al. An X-linked myopathy with postural muscle atrophy and generalized hypertrophy, termed XMPMA, is caused by mutations in FHL1. Am J Hum Genet. 2008; 82:88-99.

22. Schessl J, Zou Y, McGrath MJ, et al. Proteomic identification of FHL1 as the protein mutated in human reducing body myopathy. J Clin Invest. 2008;118:904-912.

23. Song HK, Hong SE, Kim T, et al. Deep RNA sequencing reveals novel cardiac transcriptomic signatures for physiological and pathological hypertrophy. PLoS One. 2012;7:e35552.
24. Friedrich FW, Wilding BR, Reischmann S, et al. Evidence for FHL1 as a novel disease gene for isolated hypertrophic cardiomyopathy. Hum Mol Genet. 2012; 21:3237-3254.

25. Weng J, Liao M, Zou S, et al. Downregulation of FHL1 expression in thoracic aortic dissection: implications in aortic wall remodeling and pathogenesis of thoracic aortic dissection. Ann Vasc Surg. 2011; 25:240-247.

26. Kwapiszewska G, Wygrecka M, Marsh LM, et al. Fhl-1, a new key protein in pulmonary hypertension. Circulation.2008;118:1183-1194.

27. Miele E, Tozzi A, Staiano A, et al. Persistence of abnormal gastrointestinal motility after operation for Hirschsprung's disease. Am J Gastroenterol.2000;95:1226-1230.

28. Daniel EE, Berezin I. Interstitial cells of Cajal: are they major players in control of gastrointestinal motility? J Gastrointest Motil. 1992;4:1-24.

29. Lyford GL, Farrugia G. Ion channels in gastrointestinal smooth muscle and interstitial cells of Cajal. Curr Opin Pharmacol. 2003; 3:583-587.

30. Kraichely RE, Farrugia G. Mechanosensitive ion channels in interstitial cells of Cajal and smooth muscle of the gastrointestinal tract. Neurogastroenterol Motil.2007;19:245-252.

31. Vanderwinden JM, Rumessen JJ, Liu H, et al. Interstitial cells of Cajal in human colon and in Hirschsprung's disease. Gastroenterology. 1996;111:901-910.

32. Taguchi T, Suita S, Masumoto K, et al. An abnormal distribution of C-kit positive cells in the normoganglionic segment can predict a poor clinical outcome in patients with Hirschsprung's disease. Eur J Pediatr Surg. 2005;15:153-158.

33. Leong M, Verey F, Newbury-Ecob R, et al. Supernumerary intestinal muscle coat in a patient with Hirschsprung disease/Mowat-Wilson syndrome. Pediatr Dev Pathol. 2010;13:415-418.

34. Nemeth L, Rolle U, Puri P. Altered cytoskeleton in smooth muscle of aganglionic bowel. Arch Pathol Lab Med. 2002;126:692-696.

35. Shathasivam T, Kislinger T, Gramolini AO. Genes, proteins and complexes: the multifaceted nature of FHL family proteins in diverse tissues. J Cell Mol Med. 2010;14:2702-2720.

36. Khurana T, Khurana B, Noegel AA.LIM proteins: association with the actin cytoskeleton. Protoplasma. 2002;219:1-12.

37. Shathasivam $\mathrm{T}$, Ignatchenko $\mathrm{V}$, Kislinger $\mathrm{T}$, et al. Identification of an FHL1 protein complex containing ACTN1, ACTN4, and PDLIM1 using affinity purifications and MS-based protein-protein interaction analysis. Mol Biosyst. 2011;7:1185-1196.

38. Wang L, Miao J, Li L, et al. Identification of an FHL1 Protein Complex Containing Gamma-Actin and Non-Muscle Myosin IIB by Analysis of Protein-Protein Interactions. PLoS One 2013;8:e79551. doi: 10.1371/journal.pone.0079551.

39. Yang Z, Browning CF, Hallaq H, et al. Four and a half LIM protein 1: a partner for KCNA5 in human atrium. Cardiovasc Res.2008;78:449-457.

40. Dobrev D, Wettwer E. Four and a half LIM protein 1: a novel chaperone for atrium-specific Kv1.5 channels with a potential role in atrial arrhythmogenesis. Cardiovasc Res. 2008;78:411-2.

41. Luo JL, Qin HY, Wong CK, et al. Enhanced excitability and down-regulated voltage-gated potassium channels in colonic DRG neurons from neonatal maternal separation rats. J Pain. 2011;12:600-609.

42. Liu Y, Luo H, Liang C, et al. Actions of Hydrogen Sulfide and ATP-Sensitive Potassium Channels on Colonic Hypermotility in a Rat Model of Chronic Stress. PLoS One. 2013; 8:e55853. 